\title{
Robot Assisted Training for the Upper Limb after Stroke (RATULS): study protocol for a randomised controlled trial
}

Helen Rodgers ${ }^{1,2^{*}}$, Lisa Shaw ${ }^{1}$, Helen Bosomworth ${ }^{1}$, Lydia Aird ${ }^{2}$, Natasha Alvarado ${ }^{3}$, Sreeman Andole ${ }^{4}$, David L. Cohen ${ }^{5}$, Jesse Dawson ${ }^{6}$, Janet Eyre ${ }^{7}$, Tracy Finch ${ }^{3}$, Gary A. Ford ${ }^{8,9}$, Jennifer Hislop ${ }^{10}$, Steven Hogg ${ }^{11}$, Denise Howel ${ }^{3}$, Niall Hughes ${ }^{12}$, Hermano Igo Krebs ${ }^{13}$, Christopher Price ${ }^{1,14}$, Lynn Rochester ${ }^{15}$, Elaine Stamp ${ }^{3}$, Laura Ternent ${ }^{10}$, Duncan Turner ${ }^{16}$, Luke Vale ${ }^{10}$, Elizabeth Warburton ${ }^{17}$, Frederike van Wijck ${ }^{18}$ and Scott Wilkes ${ }^{19}$

\begin{abstract}
Background: Loss of arm function is a common and distressing consequence of stroke. We describe the protocol for a pragmatic, multicentre randomised controlled trial to determine whether robot-assisted training improves upper limb function following stroke.

Methods/design: Study design: a pragmatic, three-arm, multicentre randomised controlled trial, economic analysis and process evaluation.

Setting: NHS stroke services.

Participants: adults with acute or chronic first-ever stroke ( 1 week to 5 years post stroke) causing moderate to severe upper limb functional limitation.

Randomisation groups:

1. Robot-assisted training using the InMotion robotic gym system for 45 min, three times/week for 12 weeks

2. Enhanced upper limb therapy for $45 \mathrm{~min}$, three times/week for 12 weeks

3. Usual NHS care in accordance with local clinical practice

Randomisation: individual participant randomisation stratified by centre, time since stroke, and severity of upper limb impairment.

Primary outcome: upper limb function measured by the Action Research Arm Test (ARAT) at 3 months post randomisation.

Secondary outcomes: upper limb impairment (Fugl-Meyer Test), activities of daily living (Barthel ADL Index), quality of life (Stroke Impact Scale, EQ-5D-5L), resource use, cost per quality-adjusted life year and adverse events, at 3 and 6 months.

Blinding: outcomes are undertaken by blinded assessors.

Economic analysis: micro-costing and economic evaluation of interventions compared to usual NHS care. A within-trial analysis, with an economic model will be used to extrapolate longer-term costs and outcomes.

Process evaluation: semi-structured interviews with participants and professionals to seek their views and experiences of the rehabilitation that they have received or provided, and factors affecting the implementation of the trial.

(Continued on next page)
\end{abstract}

\footnotetext{
* Correspondence: helen.rodgers@newcastle.ac.uk

${ }^{1}$ Stroke Research Group, Institute of Neuroscience, Newcastle University, 3-4

Claremont Terrace, Newcastle upon Tyne NE2 4AE, UK

${ }^{2}$ Stroke Northumbria, Northumbria Healthcare NHS Foundation Trust, North

Tyneside General Hospital, Rake Lane, North Shields, Tyne and Wear NE29

$8 \mathrm{NH}, \mathrm{UK}$

Full list of author information is available at the end of the article
} 


\begin{abstract}
(Continued from previous page)
Sample size: allowing for 10\% attrition, 720 participants provide 80\% power to detect a 15\% difference in successful outcome between each of the treatment pairs. Successful outcome definition: baseline ARAT 0-7 must improve by 3 or more points; baseline ARAT 8-13 improve by 4 or more points; baseline ARAT 14-19 improve by 5 or more points; baseline ARAT 20-39 improve by 6 or more points.
\end{abstract}

Discussion: The results from this trial will determine whether robot-assisted training improves upper limb function post stroke.

Trial registration: ISRCTN, identifier: ISRCTN69371850. Registered 4 October 2013.

Keywords: Stroke, Arm, Rehabilitation, Robotics, RCT, Cost-effectiveness analysis, Parallel process evaluation

\section{Background}

Stroke is the commonest cause of complex adult disability in high-income countries [1]. Loss of arm function affects $69 \%$ of people who have a stroke [2]. Only $12 \%$ of people with arm weakness at the onset of stroke make a full recovery [3]. Improving arm function has been identified as a research priority by stroke survivors, carers and health professionals who report that current rehabilitation pays insufficient attention to arm recovery [4].

Robot-assisted training enables a greater number of repetitive tasks to be practised in a consistent and controllable manner. Repetitive task training is known to drive Hebbian plasticity, where wiring of pathways that are coincidently active is strengthened [5, 6]. A dose of greater than $20 \mathrm{~h}$ of repetitive task training improves upper limb motor recovery following a stroke [7] and, therefore, robot-assisted training has the potential to improve arm motor recovery after stroke. We anticipate that Hebbian neuroplasticity, which is learning dependent, will operate regardless of the post-stroke phase.

A Cochrane systematic review of electromechanical and robot-assisted arm training after stroke reported outcomes from a total of 1160 patients who participated in 34 randomised controlled trials (RCTs). Improvements in arm function (standardised mean difference (SMD) 0.35, 95\% confidence interval (CI), 0.18-0.51) and activities of daily living (SMD 0.37, 95\% CI, 0.11-0.64) were found in patients who received this treatment, but studies were often of low quality [8]. In the UK there is currently insufficient evidence to justify the use of this technology in routine clinical practice.

In addition, studies which suggest that robot-assisted training may improve upper limb function after stroke should be treated with caution as participants who were randomised to receive robot-assisted training may have also received an increased intensity of rehabilitation sessions (e.g. frequency or duration) compared to participants in the control groups. Greater intensity of upper limb rehabilitation sessions has been shown to improve upper limb functional outcomes [7], and a meta-analysis of robot-assisted training RCTs reported that if control group therapy sessions were delivered at the same frequency and duration, there was no additional functional improvement [9]. Studies are required which provide further direct evidence of the effectiveness of robot-assisted training without the confounding effect of therapy dose.

The aim of the Robot Assisted Training for the Upper Limb after Stroke (RATULS) trial is to evaluate the clinical and cost-effectiveness of robot-assisted training compared to an upper limb therapy programme of the same frequency and duration, and usual post-stroke care.

The null hypothesis is that there is no difference in upper limb function at 3 months between study participants who receive robot-assisted training and those who receive an enhanced upper limb therapy programme and those who receive usual post-stroke care. The RATULS trial will be making comparisons of the effectiveness of rehabilitation on upper limb function between all three pairs of trial arms.

\section{Methods}

\section{Study aim and objectives}

Aim

To determine whether robot-assisted training with the InMotion robotic gym system (InMotion commercial version) improves upper limb function post stroke.

\section{Objectives}

- To determine whether robot-assisted training improves upper limb function post stroke compared to an enhanced upper limb therapy programme or usual care

- To determine whether robot-assisted training improves upper limb impairment, activities of daily living and quality of life compared to an enhanced upper limb therapy programme or usual care

- To model the costs of robotic-assisted training compared to an enhanced upper limb therapy programme or usual care

- To seek the views and experiences of patients and health service professionals about the upper limb rehabilitation that they have received or provided and factors affecting the implementation of the trial 
- To explore:

- the time pattern of upper limb recovery of participants in each treatment group

- the impact of the severity of baseline upper limb function and time since stroke upon the effectiveness of the interventions

\section{Study design}

This study is a three-arm, pragmatic, observer-blind, multicentre RCT with embedded economic analysis and a process evaluation. Participants are randomised to receive either: robot-assisted training (in addition to usual NHS care); an enhanced upper limb therapy programme (in addition to usual NHS care); or usual NHS care in accordance with local clinical practice. Figure 1 summarises the study methods. The study is presented according to the Standard Protocol Items: Recommendations for Interventional Trials (SPIRIT) [10] (SPIRIT Checklist, Additional file 1). Figure 2 shows the SPIRIT schedule of enrolment, interventions and assessments.

\section{Study setting}

The study is being conducted in NHS stroke units in the UK. There are four RATULS study centres (Glasgow, North Tyneside, Northwick Park, and Romford) each consisting of a hub site with an InMotion robotic gym system and spoke sites which are stroke services in adjacent trusts that refer patients to take part in the study and provide usual NHS care.

\section{Study participants}

Adults with a first-ever stroke who fulfil the following criteria are eligible to participate in the trial:

\section{Inclusion criteria}

- Age 18 years and over

- Clinical diagnosis of stroke (cerebral infarction, primary intracerebral haemorrhage, subarachnoid haemorrhage)

- Between 1 week and 5 years since stroke

- Moderate to severe upper limb functional limitation (Action Research Arm Test (ARAT) [11] score 0-39) due to stroke

- Able to provide consent to take part in the study and to comply with the requirements of the protocol

\section{Exclusion criteria}

- More than one stroke (patients with previous transient ischaemic attack (TIA) may be invited to participate)
- Other current significant impairment of the upper limb affected by stroke, e.g. fixed contracture, frozen shoulder, severe arthritis, recent fracture

- Diagnosis likely to interfere with rehabilitation or outcome assessments, e.g. registered blind

- Previous use of the InMotion robotic gym system or other arm rehabilitation robot

- Current participation in a rehabilitation trial evaluating upper limb rehabilitation after stroke

- Previous enrolment in the RATULS study

\section{Case ascertainment and recruitment}

Study participants are recruited from both incident and prevalent stroke populations. Participants can be sought from a number of settings in both primary and secondary care including: stroke units; outpatient clinics; day hospitals; community rehabilitation services; and general practices. The study aims to recruit similar numbers of participants within: 0-3 months of stroke; >3-12 months after stroke; and $>12$ months to 5 years after stroke.

\section{Potential participants from secondary care}

In secondary care, potential participants are identified by local clinicians and/or staff from the National Institute for Health Research Local Clinical Research Network (NIHR LCRN). Staff approach potentially eligible patients, discuss the study and provide a study information leaflet. After allowing sufficient time for the information to be considered, staff ask the patient if they are potentially interested in taking part in the study.

Potential participants can also be identified from hospital stroke discharge summaries/clinic letters. If this method is used, potential participants are approached by letter. Enclosed with the letter is a short RATULS leaflet, a Patient Information Sheet, a RATULS reply slip and a pre-paid envelope. Interested patients may make contact with the study centre by telephone or by return of the RATULS reply slip. Following a few short telephone questions to confirm potential study eligibility, a face-toface appointment for further discussion is subsequently arranged if appropriate.

\section{Potential participants from primary care}

To identify potential participants from primary care, general practices perform a database search using the study inclusion/exclusion criteria. A GP screens the list of potentially eligible participants to approve the issue of an invitation letter. This letter is accompanied by the same information which is sent to individuals identified from secondary care records. The invitation letter details the main study eligibility criteria and asks interested patients to contact the study centre for further information. Following a few short telephone questions to confirm 


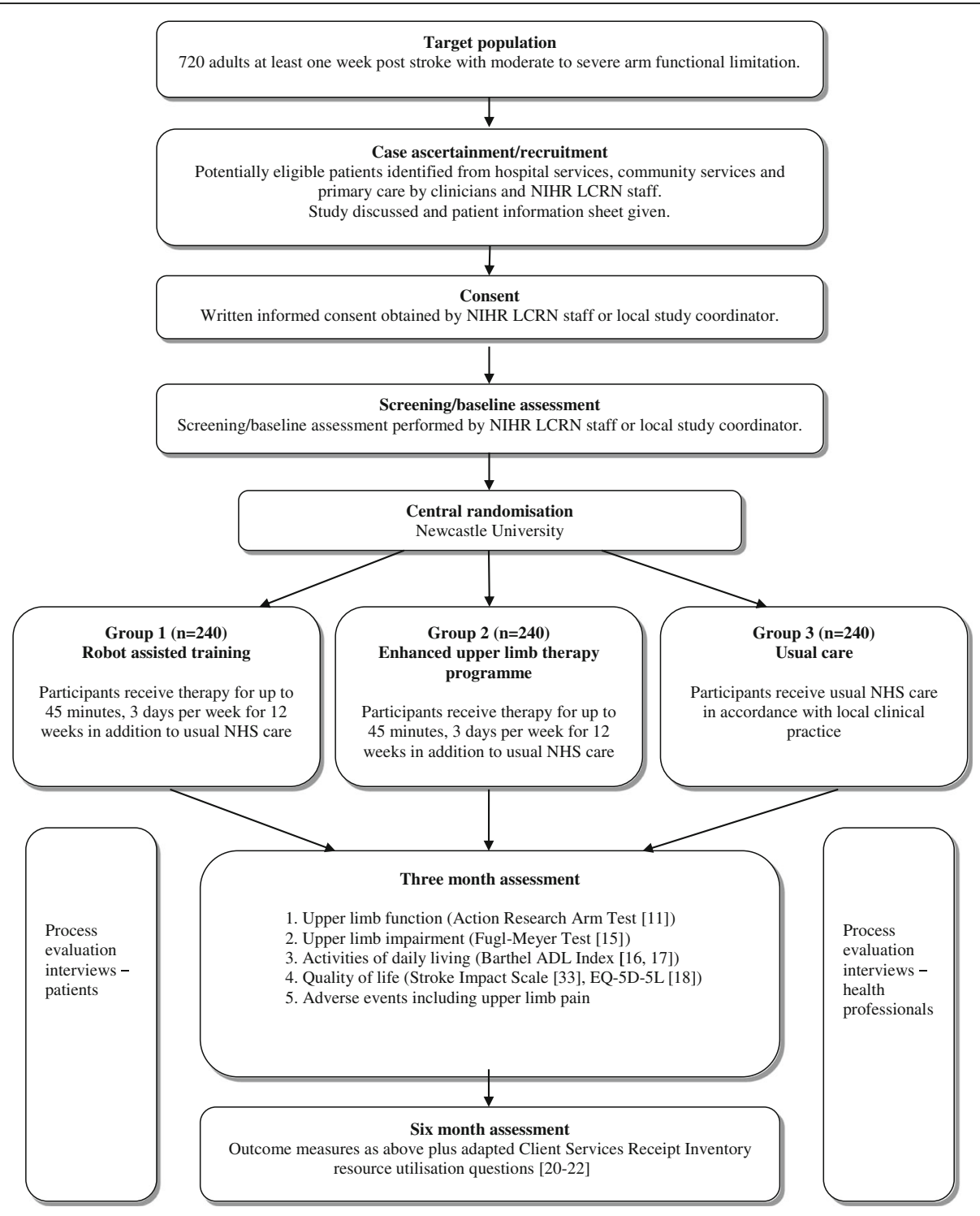

Fig. 1 RATULS trial summary

potential study eligibility, a face-to-face appointment for further discussion is subsequently arranged if appropriate.

\section{Potential patients from other sources}

Local community stroke clubs and day centres are also given information about the study. In addition, some individuals may hear about the study from press releases or see information about the study on a poster or RATULS leaflet. Interested individuals are able to contact the study centres directly for a discussion about the study.

\section{Consent}

Individuals who are interested and potentially eligible to take part in the study are given an appointment for further discussion and consent. This may be conducted by a local study coordinator or NIHR LCRN staff. Written informed consent is obtained if the patient wishes to take part in RATULS.

\section{Screening log}

A screening log is kept at each study centre. This records details of all inpatients, outpatients and primary care patients considered for the study and subsequently included or excluded.

\section{Screening assessment}

Once written informed consent is obtained, a screening assessment is performed by the local study centre coordinator or NIHR LCRN staff. The following data are 


\begin{tabular}{|c|c|c|c|c|c|}
\hline & & \multicolumn{4}{|c|}{ Study period } \\
\hline & & \multirow{2}{*}{$\begin{array}{l}\text { Enrolment } \\
\text { Screening }\end{array}$} & \multirow{2}{*}{$\begin{array}{l}\text { Allocation } \\
\text { Baseline }\end{array}$} & \multicolumn{2}{|c|}{ Post-allocation } \\
\hline & & & & 3 months & 6 months \\
\hline \multicolumn{2}{|c|}{ Assessment/ activity } & $-t_{1}$ & 0 & $t_{1}$ & $t_{2}$ \\
\hline \multirow{6}{*}{$\begin{array}{l}\text { Screening } \\
\text { assessment }\end{array}$} & Written informed consent & $\mathrm{x}$ & & & \\
\hline & Contact details & $x$ & & & \\
\hline & Demography & $\mathrm{x}$ & & & \\
\hline & Stroke details & $x$ & & & \\
\hline & Comorbidity & $\mathrm{x}$ & & & \\
\hline & $\begin{array}{l}\text { Upper limb function (Action } \\
\text { Research Arm Test [11]) }\end{array}$ & $\mathrm{x}$ & & & \\
\hline \multirow{11}{*}{$\begin{array}{l}\text { Baseline } \\
\text { assessment }\end{array}$} & $\begin{array}{l}\text { Stroke severity (National Institute for } \\
\text { Health Stroke Scale [12]) }\end{array}$ & & $x$ & & \\
\hline & $\begin{array}{l}\text { Cognition function (Montreal } \\
\text { Cognitive Assessment [13]) }\end{array}$ & & $x$ & & \\
\hline & $\begin{array}{l}\text { Language skills (Sheffield Aphasia } \\
\text { Screening Test [14]) }\end{array}$ & & $x$ & & \\
\hline & $\begin{array}{l}\text { Upper limb impairment (Fugl-Meyer } \\
\text { motor and sensory arm sections } \\
{[15] \text { ) }}\end{array}$ & & $x$ & & \\
\hline & $\begin{array}{l}\text { Activities of daily living (Barthel ADL } \\
\text { Index }[16,17])\end{array}$ & & $\mathrm{x}$ & & \\
\hline & Quality of life (EQ-5D-5L [18]) & & $\mathrm{x}$ & & \\
\hline & $\begin{array}{l}\text { Upper limb pain (numerical rating } \\
\text { scale [19]) }\end{array}$ & & $x$ & & \\
\hline & $\begin{array}{l}\text { Current upper limb rehabilitation } \\
\text { treatments }\end{array}$ & & $x$ & & \\
\hline & Randomisation & & $x$ & & \\
\hline & $\begin{array}{l}\text { Pre-study resource utilisation } \\
\text { (adaptation of the Client Services } \\
\text { Receipt Inventory [20-22]) }\end{array}$ & & $x$ & & \\
\hline & Issue of study arm rehabilitation logs & & $\mathrm{x}$ & & \\
\hline \multirow{3}{*}{ Interventions } & $\begin{array}{l}\text { Robot-assisted training in addition to } \\
\text { usual care }\end{array}$ & & & $\rightarrow$ & \\
\hline & $\begin{array}{l}\text { Enhanced upper limb therapy in } \\
\text { addition to usual care }\end{array}$ & & & $\rightarrow$ & \\
\hline & Usual care & & 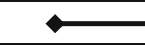 & $\rightarrow$ & \\
\hline \multirow{10}{*}{$\begin{array}{l}\text { Outcome } \\
\text { assessments }\end{array}$} & $\begin{array}{l}\text { Upper limb function (Action } \\
\text { Research Arm Test [11]) }\end{array}$ & & & $x$ & $\mathrm{x}$ \\
\hline & $\begin{array}{l}\text { Upper limb impairment (Fugl-Meyer } \\
\text { motor and sensory arm sections } \\
\text { [15]) }\end{array}$ & & & $x$ & $\mathrm{x}$ \\
\hline & $\begin{array}{l}\text { Activities of daily living (Barthel ADL } \\
\text { Index }[16,17])\end{array}$ & & & $\mathrm{x}$ & $\mathrm{x}$ \\
\hline & Quality of life (EQ-5D-5L [18]) & & & $x$ & $x$ \\
\hline & $\begin{array}{l}\text { Impact of stroke } \\
\text { (Stroke Impact Scale [33]) }\end{array}$ & & & $x$ & $\mathrm{x}$ \\
\hline & $\begin{array}{l}\text { Upper limb pain (numerical rating } \\
\text { scale [19]) }\end{array}$ & & & $\mathrm{x}$ & $\mathrm{x}$ \\
\hline & Adverse Events & & & $x$ & $x$ \\
\hline & $\begin{array}{l}\text { Resource utilisation (adaptation of } \\
\text { the Client Services Receipt Inventory } \\
\text { [20-22]) }\end{array}$ & & & & $x$ \\
\hline & $\begin{array}{l}\text { Time and travel resource utilisation } \\
{[34,35]}\end{array}$ & & & & $\mathrm{x}$ \\
\hline & Issue of study arm rehabilitation logs & & & $\mathrm{x}$ & \\
\hline
\end{tabular}

Fig. 2 Standard Protocol Items: Recommendations for Interventional Trials (SPIRIT) schedule of enrolment, interventions and assessments

collected: demography; stroke details; comorbidity; and upper limb function (ARAT score [11]). If the patient fulfils the study inclusion and exclusion criteria, the local study coordinator/NIHR LCRN staff proceeds to the baseline assessment. If it is not possible to complete the baseline assessment on the same day as the screening 
assessment, eligibility for the study is re-confirmed on the day of the baseline assessment.

\section{Baseline assessment}

The following baseline data are collected: stroke severity (National Institute for Health Stroke Scale [12]); cognitive function (Montreal Cognitive Assessment [13]); language skills (Sheffield Aphasia Screening Test [14]); upper limb impairment (Fugl-Meyer Test (motor and sensory arm sections) [15]); activities of daily living (Barthel ADL Index [16, 17]; quality of life (EQ-5D-5 L [18]); upper limb pain (numerical rating scale [19]) and current upper limb rehabilitation treatments. In addition, patients are given a self-completion questionnaire containing pre-study resource utilisation questions (adaption of the Client Services Receipt Inventory [20-22]) which they are asked to complete at the end of the assessment.

\section{Randomisation}

Randomisation is conducted by the local study coordinator/NIHR LCRN staff following completion of the baseline assessment. A central independent web-based service hosted by Newcastle University Clinical Trials Unit is used. Participants are stratified according to study centre, time since stroke and severity of upper limb function (ARAT score [11]), and randomised to either robotassisted training, enhanced upper limb therapy, or usual care groups using permuted block sequences.

\section{Randomisation groups \\ Robot-assisted training using the InMotion robotic gym system}

This is delivered using the InMotion robotic gym system which was specifically designed for clinical rehabilitation applications [23-25]. This is currently the most widely used technology for robot-assisted training for patients with moderate to severe upper limb impairment post stroke. The system development started in 1989 and it has amassed the largest body of clinical evidence to date of any robotic system. It has been successfully tested in clinical studies involving over 900 stroke patients and there are around 250 robots in use worldwide.

Participants receive robot-assisted training at the hub sites for up to $45 \mathrm{~min}$ per day, three days per week for 12 weeks, in addition to usual care. A detailed description of the robot-assisted training programme using the Template for Intervention Description and Replication (TIDieR) Checklist [26] is provided in Table 1.

\section{Enhanced upper limb therapy programme}

The enhanced upper limb therapy programme aims to match the frequency and duration of the robot-assisted training programme sessions. It has been developed from the upper limb therapy programmes used in the
Botulinum Toxin for the Upper Limb after Stroke (BoTULS) trial [27-29] and the Repetitive Arm Functional Tasks after Stroke (RAFTAS) project [30]. Using the principles of person-centred goal-setting and repetitive functional task practice, it aims to drive neuroplasticity and motor recovery after stroke.

Participants receive enhanced upper limb therapy at the hub sites for up to $45 \mathrm{~min}$ per day, 3 days per week for 12 weeks, in addition to usual care. A detailed description of the enhanced upper limb therapy programme using the TIDieR Checklist [26] is provided in Table 1.

\section{Usual care}

Defining usual care is a challenge for any stroke rehabilitation trial. One of the current NICE quality standards is that 'patients with stroke should be offered a minimum of $45 \mathrm{~min}$ of each appropriate therapy that is required, for a minimum of 5 days a week, at a level that enables the patient to meet their rehabilitation goals for as long as they are continuing to benefit from therapy and as long as they are able to tolerate it' [31]. For most stroke services this is aspirational and the majority of patients do not receive this intensity particularly after discharge from hospital or early supported discharge services [32]. Patients with chronic stroke are unlikely to receive ongoing rehabilitation in the longer term. Most services do not regularly review patients to address unmet rehabilitation needs beyond 1 year.

Usual care is delivered at hub and spoke sites.

Participants in all three randomisation groups receive a study 'arm rehabilitation therapy log' where they are asked to record any 'usual' upper limb rehabilitation that they receive during the course of the study. Periodic text message reminders are sent to remind participants about completion of the rehabilitation logs. In addition, participants in all three randomisation groups receive regular study newsletters.

\section{Outcome assessments}

Outcomes are assessed at 3 months ( \pm 7 days) and 6 months ( \pm 7 days) following randomisation.

Assessments are undertaken in two stages:

Stage 1 is a self-completion postal questionnaire consisting of the Stroke Impact Scale (SIS) [33] (3 and 6 months) and the adapted Client Services Receipt Inventory resource utilisation questions (6 months only) [20-22].

Stage 2 is a face-to-face assessment with a researcher blinded to randomisation group. The following data are collected: Barthel ADL Index [16, 17], EQ-5D-5L [18], ARAT [11], Fugl-Meyer Test (motor and sensory arm sections [15]), and adverse events. At the end of the 6-month stage-2 assessment, participants are given a further selfcompletion questionnaire and are asked to return this by 


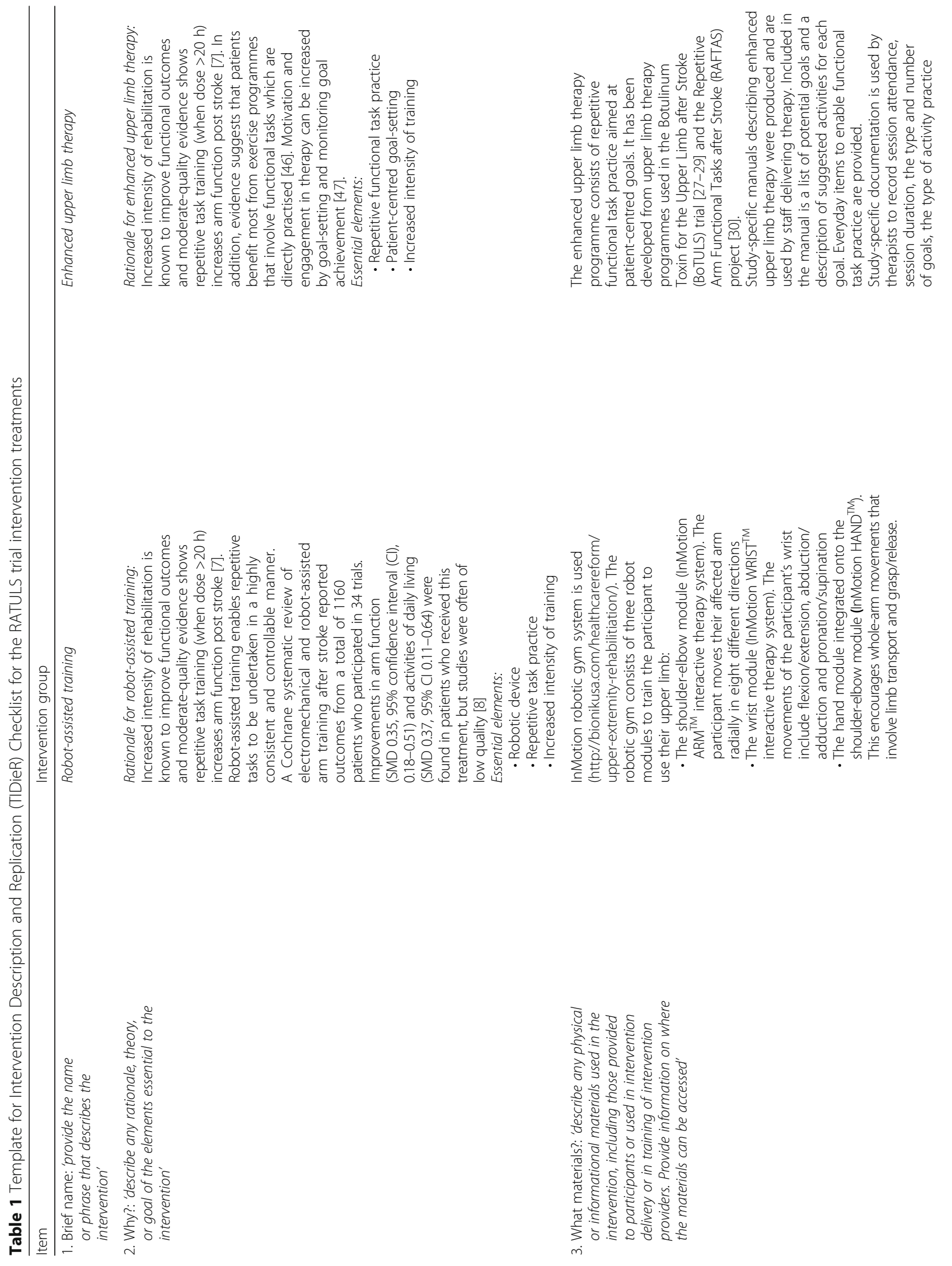




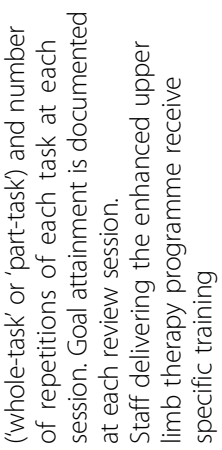
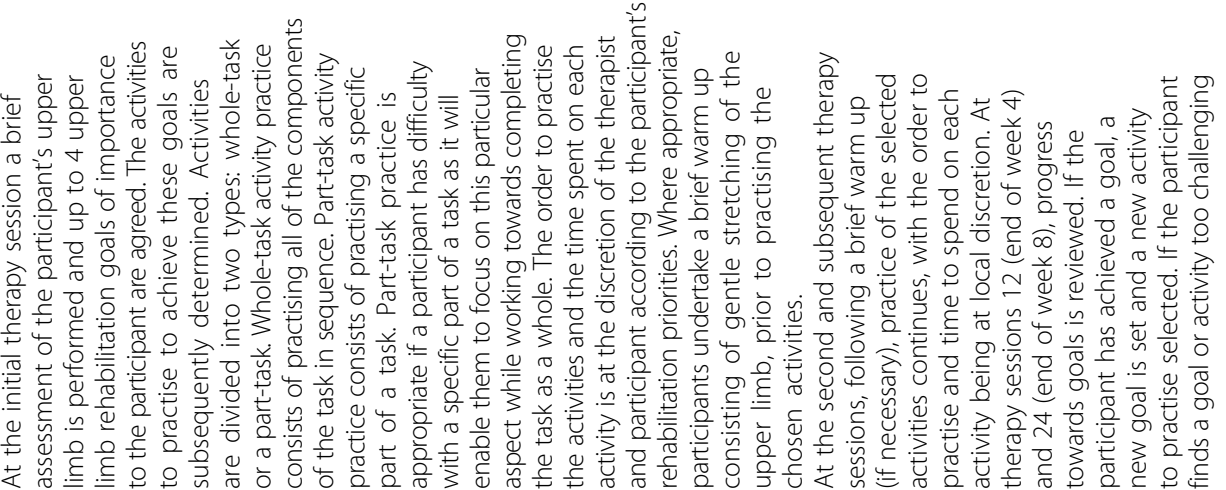

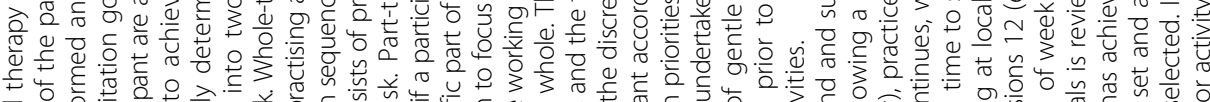

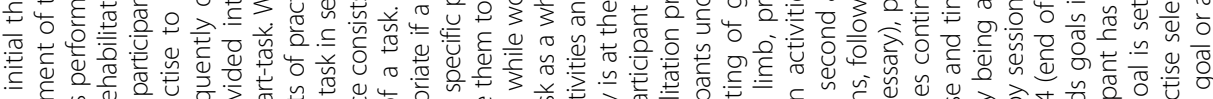

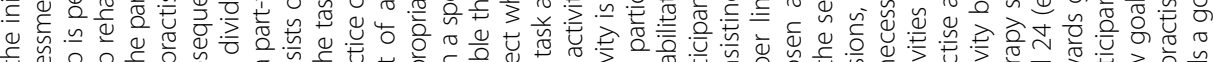

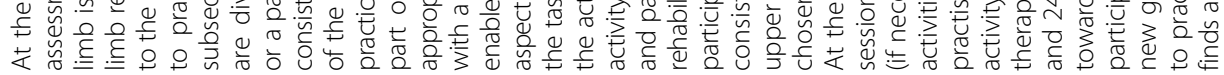

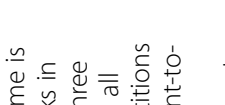

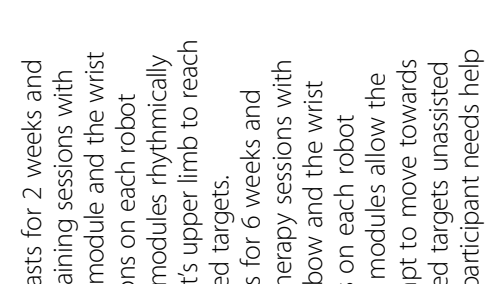

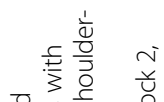

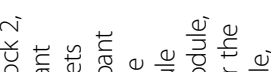




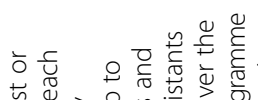

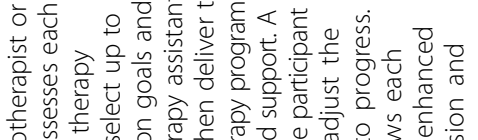

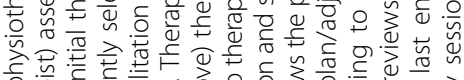

$\stackrel{0}{\stackrel{2}{Q}}$

⿶凵

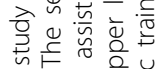

作

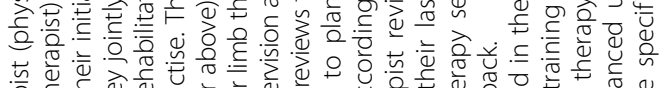

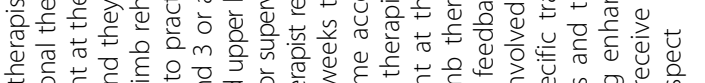

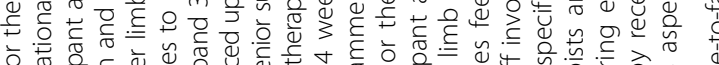

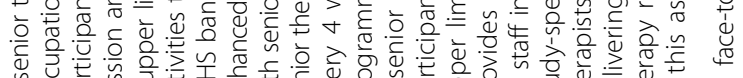

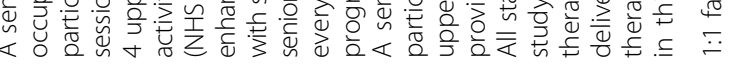

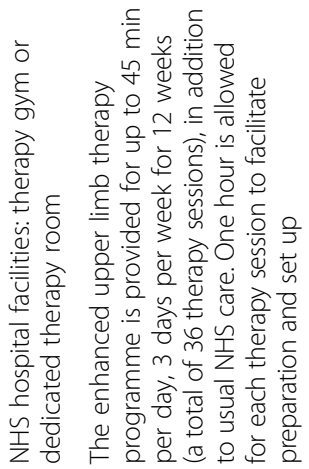

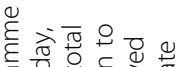

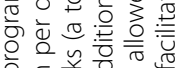

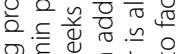

들

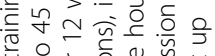

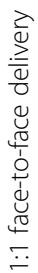
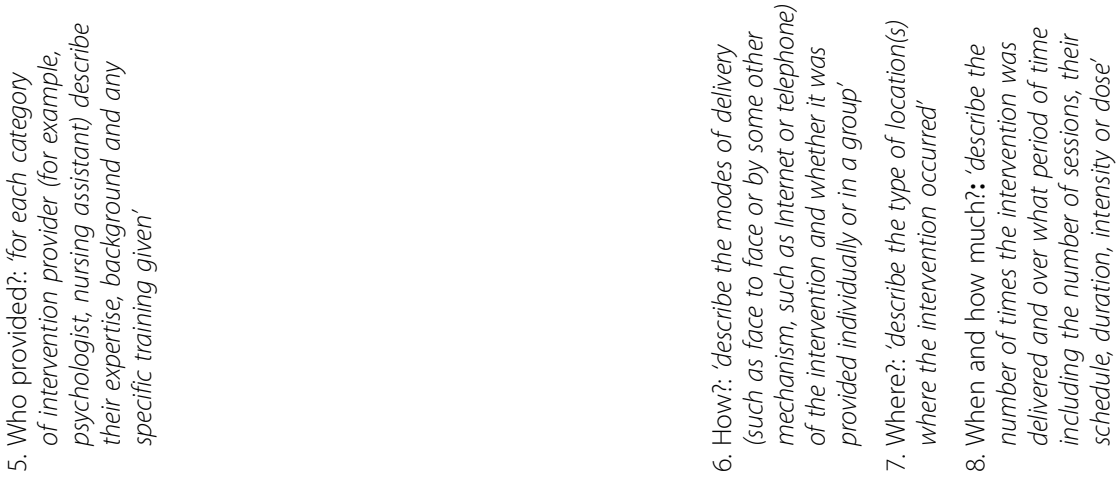


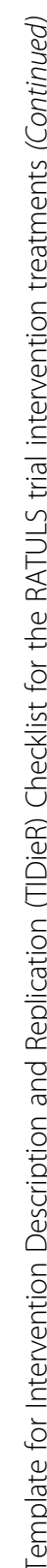

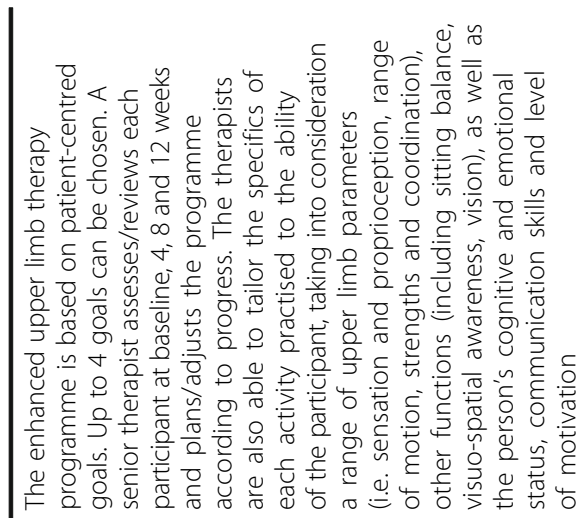

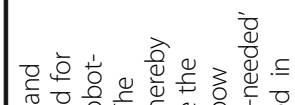

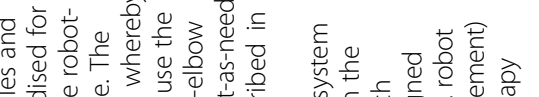

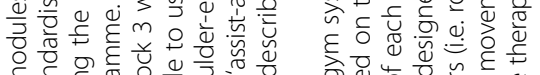

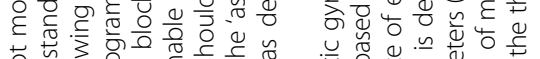

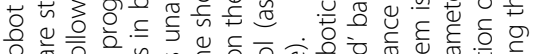

等

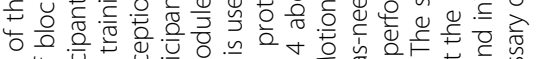

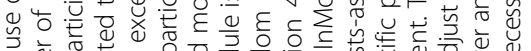

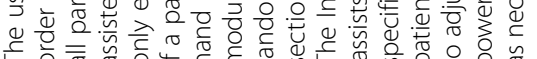

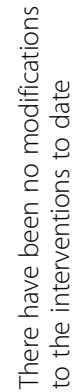

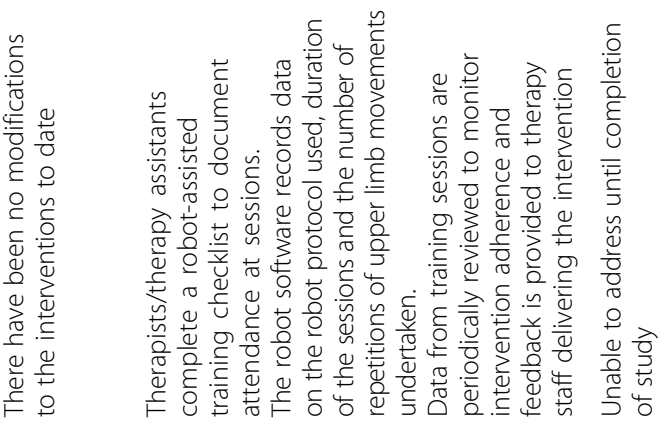

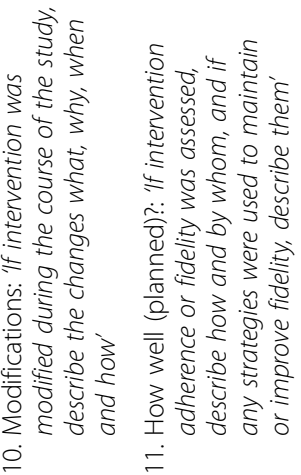

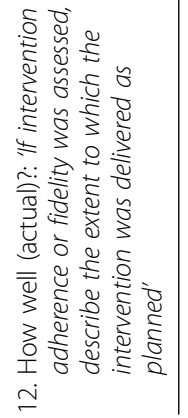


post. This questionnaire contains time and travel resource use questions $[34,35]$.

\section{Blinding}

Due to the nature of the interventions, it is not possible to blind participants or treating therapists to treatment allocation. It is intended that stage- 2 outcome assessments are conducted by a researcher blinded to treatment allocation. After each outcome assessment the researcher is asked to record whether they have unintentionally become aware of treatment allocation due to conversation with the participant. Success of outcome assessment blinding will be reported.

\section{Study withdrawal}

No specific withdrawal criteria have been pre-set. Participants may withdraw from the study at any time for any reason. Data collected prior to withdrawal will be used in the study analysis unless consent for this is specifically withdrawn. Should a decision to withdraw from the study be made, a reason for withdrawal is sought but participants can chose to withdraw without providing an explanation.

Investigators, GPs, stroke physicians and therapists may also withdraw participants from the study at any time if they feel it is no longer in their interest to continue; for example, because of intercurrent illness or adverse events.

\section{Safety evaluation}

The safety of robot-assisted training, enhanced upper limb therapy and usual care is being evaluated by examining the occurrence of all adverse events and serious adverse events in accordance with National Research Ethics Committee (NRES) guidance for non-CTIMP trials [36].

\section{Statistical analysis Primary analysis}

The primary outcome is arm function measured by the ARAT [11] at 3 months. It has been suggested that the minimal clinically important difference for the ARAT is $10 \%$ of its range ( 6 points) [37] but a smaller treatment effect may be clinically beneficial in those with severe initial upper limb functional limitation who are likely to improve less than those with more moderate limitation. There will be a stepped approach to define 'successful outcome': baseline ARAT $0-7$ must improve by 3 or more points; baseline ARAT 8-13 improve by 4 or more points; baseline ARAT 14-19 improve by 5 or more points; baseline ARAT 20-39 improve by 6 or more points. Analyses will be by intention-to-treat. Logistic regression will be used to compare the primary outcome (success) between the three randomisation groups at 3 and 6 months, adjusting for any imbalance in key covariates. The use of multilevel logistic models will be explored. It may be possible to fit three-level models (hubs, spokes and participants), but since there are only four centres with a hub, and a small number of stroke services accessing an InMotion robotic gym system at each hub, it may be necessary to fit a two-level model (stroke services and participants).

\section{Secondary analyses}

The secondary outcomes will be compared between the three groups at 3 and 6 months using multilevel linear regression adjusting for baseline values and key covariates.

We will consider any difference in attrition rates, and any nonrandomness of the attrition, when comparing outcomes between the three groups. The pattern of missing observations because of loss to follow-up will be examined to determine both the extent of missingness, and whether it is missing at random or is informative. If data are missing to a sufficient extent, the use of appropriate multiple imputation techniques will be considered. Although mortality is possible within the 6-month follow-up period, it is thought to be sufficiently uncommon that methods for joint modelling of survival and longitudinal data will not be necessary.

Further descriptive analyses will explore the relationship between the severity of baseline upper limb function and time since stroke upon the effectiveness of the intervention. There is not sufficient power to perform any formal subgroup analyses. The time pattern of upper limb recovery will be explored by extending the earlier multilevel models to include a further within-patient level (ARAT scores collected at baseline, 3 and 6 months). However, this will depend on the relationship being approximately linear.

\section{Sample size}

The sample size is 720 participants ( 240 participants per group). Responses from 216 participants in each randomisation group will provide $80 \%$ power (significance level of $1.67 \%$ because of multiple comparisons) to detect a $15 \%$ difference in 'successful outcome' between each of the three pairs of treatments (robot-assisted training, enhanced upper limb therapy, usual care). We have allowed for $10 \%$ attrition and inflated the sample size to 720 participants.

\section{Economic analysis}

The economic analysis will include a detailed microcosting analysis, economic evaluation and a longer-term economic model. This will be based upon both a 'within trial' analysis and a modelling exercise to explore costs and effects over the longer term. Analyses will be carried out from the perspective of the NHS and personal and 
social services, but we will also take a societal perspective by including costs borne by the participants and their informal carers. All relevant costs associated with providing the interventions will be measured, this will include the cost of using the InMotion robotic gym system, costed on a per patient basis. All costs will be derived using routine data sources [38] and studyspecific estimates. Where appropriate, discounting will be applied to costs and outcomes [39]. Costs in the follow-up period will also be taken into account; this includes secondary care resource, e.g. inpatient stays and outpatient visits; primary care resource use, e.g. general practice, therapy visits and prescription costs. These data will be collected using a health service utilisation questionnaire (adaption of the Client Services Receipt Inventory [20-22]) administered at baseline and 6 months post randomisation. Patient costs will also be collected via a time-and-travel questionnaire based upon one successfully used in a number of previous NIHR HTAfunded trials $[34,35]$. This will include questions relating to travel time, time away from employment (if appropriate) and time spent providing care. The within-trial analysis will also compare changes in health-related quality of life, based on responses to the EQ-5D-5L at baseline, 3 and 6 months post randomisation and scored using population tariffs [40]. These data will be combined with study participants' mortality to estimate quality-adjusted life years (QALYs). This measure provides a profile of quality of life over time. The results of the analyses will be presented as point estimates of mean incremental costs and QALYs. Techniques, such as bootstrapping, will be used alongside deterministic sensitivity analyses to address uncertainty [41]. In addition, a within trial costutility analysis will be performed where both costs and QALY data will be combined into an incremental cost per QALY. The cost-utility analysis will include deterministic and stochastic sensitivity analysis, presented as point estimates and cost-effectiveness acceptability curves (CEACs).

An economic model will also be developed to assess the cost and health consequences measured in terms of QALYs of stroke recovery beyond the 6-month timeframe of the trial. The data from the trial will be the main source of data for this model but further data with which to model outcomes beyond a 6-month follow-up will be systematically derived from the academic literature and other existing data sources following guidance for best practice [42]. These data will include information on factors, such as the incidence of hospitalisation and the need for residential/nursing home care, beyond the trial follow-up period. Sensitivity analysis will be applied to the model using probabilistic and deterministic sensitivity analyses to address parameter and other forms of uncertainty. The data on both costs and QALYs for both trial- and modelbased analyses will be reported separately.

\section{Parallel process evaluation}

Alongside the RCT, a two-stage process evaluation is being conducted to understand both (1) participants' and health service professionals' experiences of robot-assisted training; enhanced upper limb therapy and usual care and (2) factors affecting the implementation of the trial within and across study sites. The process evaluation will capture data concerning feasibility and accumulating experience of the therapies being provided. In stage 1 data collection is by semi-structured interview using a pre-developed and piloted interview schedule. Data collection in stage 2 is primarily by interview; however, analysis also draws upon trial data including baseline, therapy and outcome (3 and 6 month) assessments. Interviews are primarily being conducted face to face; however, due to the geographical spread of the study sites, some follow-up interviews are being conducted by telephone for efficiency (these are particularly appropriate for health service professionals). Data collection and analysis relating to study of implementation factors will be informed by Normalization Process Theory (NPT) [43].

\section{Participant study group}

In stage 1 a subset of approximately 25-30 study participants will be recruited across study sites, to achieve a maximum variation sample, ensuring representation of participants differing in terms of key factors such as randomisation group, clinical severity and time from stroke. Participants in the robot-assisted training and enhanced upper limb therapy programme groups are interviewed on two occasions: (1) soon after therapy commences and (2) towards the end of the 12-week therapy period, to determine how perceptions of acceptability of therapy may change over time.

In stage 2 approximately 25 participants will be recruited, again with the aim of achieving maximum variation in the sample. Participants in the treatment groups are interviewed twice. However, in this stage, time points are (1) towards the end of their 12-week therapy and (2) around their 6-month follow-up assessments, to provide insight into their experience of trial participation, and the impact of the therapy they received, post treatment. The baseline, therapy and outcome assessment data are reviewed descriptively, for the participants who have been interviewed as part of stage 2. This will allow comparison of trial participants' assessment data with their subjective experiences of participating in the trial, to inform later interpretation of the results of the trial.

Participants to be invited for interview are identified from the study database (containing data held by unique study number only) by the researcher conducting the interviews. The researcher advises the local study centre coordinators/administrators of the selected participant numbers and the local study coordinator/administrator/ 
LCRN staff mails an invitation letter, an Information Sheet and a self-completion Contact Details Form for the participant to return directly to the researcher if they are interested in taking part in the interview(s).

The researcher telephones the responding participants, describes the purpose of the interview(s) and agrees a mutually convenient time for a first interview to take place. Prior to any potential second interview, participants are re-contacted by the researcher to check that they are still willing to take part in the second interview. Consent to be interviewed is obtained in writing prior to commencement of each interview.

\section{Health service professional study group}

A sample of approximately 20 health service professionals is being recruited across study sites and study groups. Interviews take place in stage 1 and stage 2 of the process evaluation. The aim is to interview a range of health service professionals, e.g. senior therapists, therapy assistants, study administrators, principal investigators and NIHR LCRN staff to gain insight into different aspects of the trial including implementation of the robot-assisted training, enhanced upper limb therapy and usual care practices, and implementation of the trial itself, including the recruitment and follow-up processes.

Staff to be invited for interview are identified by the local study centre coordinator and/or local study investigators. Each selected member of staff receives a letter of invitation and an Information Sheet. Following issue of the invitation letter and Information Sheet, the researcher conducting the interviews contacts the selected staff to go over the purpose of the interviews and ascertain willingness to take part. A mutually convenient time and place for the interview(s) is agreed. Consent to be interviewed is obtained in writing prior to commencement of each interview.

\section{Interview data analysis}

Interviews are audio-taped with the respondents' consent, and transcribed. Data will be mostly analysed using the constant comparative method of qualitative analysis [44] facilitated by analysis software (QSR NVivo). For a subset of the process evaluation data that specifically focussed on questions concerning implementation - a theory-based approach to analysis will be undertaken [43]. All data analysis will include a proportion of data to be analysed collectively in 'data clinics' where the research team share and exchange interpretations of key themes emerging from the data. A larger proportion of data, however, will be independently thematically coded and compared between two researchers to ensure consistency in the interpretation of data within a broader thematic framework developed as data collection progresses.

\section{Confidentiality}

Personal data are regarded as strictly confidential. Original paper Case Record Forms containing study data are stored in the investigator site file at each research site. All study files are securely stored and access restricted to staff involved in the study. Research staff at sites enter data from paper forms onto a secure web-based electronic database run and maintained by Newcastle University. Data are entered using participant-unique study numbers only. Access to this database is password-protected and limited to staff at research sites or Newcastle University who are involved in the study.

The InMotion robotic gym computers store data from each participant session. Data are stored by unique study number only. Periodically, these data are copied from the robot computer system into an electronic database maintained by Newcastle University.

The study complies with the Data Protection Act 1998, and Caldicott Guardian approval for use of patient identifiable data.

\section{Trial monitoring, quality control and quality assurance}

The chief investigator has overall responsibility for study conduct. The principal investigators are responsible for the day-to-day study conduct at their individual sites.

The trial is managed by a coordinating centre based at Newcastle University which provides day-to-day support for the sites and provides training through investigator meetings, site initiation visits and routine monitoring visits. A Trial Management Group (TMG) has been convened and the TMG meets regularly during the study.

Quality control is maintained through adherence to Newcastle Biomedicine Clinical Research Platform SOPs, the study protocol and research governance regulations. General monitoring of study conduct and data collected is being performed by a combination of central review and site-monitoring visits. The main areas of focus include consent, serious adverse events and essential documents in study files. All monitoring findings are reported and followed up with the appropriate persons in a timely manner.

A Trial Steering Committee (TSC) has been convened. This comprises an independent chair, three other independent members, a patient and/or a carer representative and the chief investigator. The TSC has agreed a charter of operation and meet at least annually.

An independent Data Monitoring and Ethics Committee (DMEC) has been convened to undertake independent review. This comprises five independent members including expert health care professionals and a statistician. Only the DMEC has access to unblinded outcome data before the trial ends. The DMEC has agreed a charter of operation and meets at least annually. 


\section{Dissemination of results}

The data are the property of the chief investigator and co-investigator(s). Publication will be the responsibility of the chief investigator.

The study will be presented at national and international conferences, and reported in peer-reviewed journals and a NIHR HTA monograph. Reports will be written for the study sponsor and regulatory bodies. A summary of the results will be sent to study participants and be available on the study website: https://research.ncl.ac.uk/ratuls/.

Anonymised data will be provided to research databases as requested (e.g. the Cochrane Collaboration, the Virtual International Stroke Trials Archive (VISTA)) to enable future meta-analyses. Anonymised robot kinematic and kinetic data will be provided to co-investigators for exploratory analyses.

\section{Discussion}

Robot-assisted training is a promising treatment for improving the upper limb function of patients with moderate to severe upper limb impairment post stroke, but further high-quality research is needed before this technology should be integrated into clinical practice [8].

RATULS is a large, multicentre RCT to determine whether robot-assisted training with the InMotion robotic gym system (MIT-Manus commercial version) improves upper limb function post stroke when compared to an upper limb therapy programme of the same frequency and duration of sessions and usual NHS care. The results from the trial will inform clinicians and commissioners of health care about the clinical effectiveness and costeffectiveness of robot-assisted training.

\section{Trial status}

RATULS commenced recruitment in April 2014. Four NHS study centres (Glasgow, North Tyneside, Northwick Park, and Romford) are participating. The RATULS trial has recruited 468 patients at the time of submission of this manuscript. Protocol version 3, dated 2 August 2016, was used to prepare the manuscript.

\section{Additional file}

Additional file 1: SPIRIT Checklist for RATULS protocol paper. (DOC $120 \mathrm{~kb}$ )

\section{Abbreviations}

ADL: Activities of daily living; ARAT: Action Research Arm Test; CEA: Costeffectiveness analysis; CEAC: Cost-effectiveness acceptability curve; CSRI: Client Services Receipt Inventory; GP: General practitioner; LCRN: Local Clinical Research Network; NHS: National Health Service; NICE: National Institutes for Health and Care Excellence; NIHR: National Institutes for Health Research; NIHR HTA: National Institutes for Health Research Health Technology Assessment; NIHSS: National Institutes of Health Stroke Scale; NRES: National Research Ethics Service; QALY: Quality-adjusted life year;
RCT: Randomised controlled trial; SIS: Stroke Impact Scale; UK: United Kingdom

\section{Acknowledgements}

We would like to thank the following for their contribution:

1. Patients taking part in the trial.

2. Staff at RATULS NHS study centres: Glasgow study centre: Jen Alexander,

Elizabeth Colquhoun, Ozlem Dincarslan, Wendy Jackson, Pamela

MacKenzie, Karen Shields and staff at surrounding spoke sites. North

Tyneside study centre: Rebecca Davidson, Judith Murdy, Victoria Riddell, Anna Smith, Leanne Smith, Gail Storey, Marie Twentyman, Helen Walker, Rebecca Watson and staff at surrounding spoke sites. Northwick Park study centre: Aberami Chandrakumar, Anette David, Fenglin Guo, Mushiya Mpelembue, Anne Oshodi, Nadine Ryan and staff at surrounding spoke sites. Romford study centre: Karen Dunne, Janice Hastings, Louise Hinkins, Rabiya Patel, Sam King and staff at surrounding spoke sites.

3. Staff at Newcastle University who contribute to the project: Richard Francis, Michael Adams.

4. Data Monitoring and Ethics Committee members: John Bamford, Richard McManus, Rebecca Palmer, Philip Rowe, Christopher Weir.

5. Trial Steering Committee members: Jennifer Burr, Jane Burridge, Nikola Sprigg, Sarah Tyson, Judith Williamson.

6. The National Institute for Health Stroke Research Network and the National Institute for Health Research (NIHR) Clinical Research Network (CRN) for Stroke.

\section{Funding}

This project is funded by the NIHR Health Technology Assessment programme (project number 11/26/05). The views and opinions expressed herein are those of the authors and do not necessarily reflect those of the HTA programme, the NIHR, the NHS or the Department of Health. The NIHR advised on the study design but had no role in writing this manuscript or the decision to submit for publication.

Availability of data and materials

Not applicable.

\section{Authors' contributions}

HR, LS, LA, JD, JE, TF, GAF, SH, DH, NH, HIK, CP, LR, LT, DT, LV, EW, FVW and SW were involved in the study design and application for funding. $H R$ is the chief investigator. LS and HB manage the trial. TF and NA lead on the parallel process evaluation. LT, JH and LV lead on the health economics. $\mathrm{DH}$ and ES lead on the statistical analysis. HIK and DT provide advice on the robot-assisted training programme. LA and FVW provide advice on the enhanced upper limb therapy programme. JD, NH, SA, DLC and HR are study centre principal investigators. LA, SA, DLC, JD, JE, GAF, SH, NH, CP, LR, FVW and SW provide advice on stroke rehabilitation and clinical trial management. All authors have commented upon drafts of the manuscript and have given final approval to this version.

\section{Ethics approval and consent to participate}

The study sponsor is Newcastle upon Tyne Hospitals NHS Foundation Trust The study is being conducted in accordance with Research Governance Framework for Health and Social Care [45]. Ethical approval was granted by the National Research Ethics Committee Sunderland (reference: 13/NE/0274). NHS Trust approvals have also been granted from Northumbria Healthcare NHS Foundation Trust, NHS Greater Glasgow and Clyde, Barking, Havering and Redbridge University Hospitals NHS Trust and London North West Hospitals NHS Trust. The following have NHS Trust approvals to act as spoke sites (Participant Identification Centres): Newcastle upon Tyne Hospitals NHS Foundation Trust, South Tyneside NHS Foundation Trust, Gateshead Health NHS Foundation Trust, County Durham and Darlington NHS Foundation Trust, North Middlesex University Hospital NHS Trust, University College London Hospitals NHS Foundation Trust, Barts Health NHS Foundation Trust, Mid Essex Hospital Services NHS Trust, North East London Foundation Trust and Royal Free London NHS Foundation Trust.

Written informed consent is obtained for all RATULS participants.

Consent for publication

Not applicable. 


\section{Competing interests}

Dr. HI Krebs is a co-inventor in the MIT-held patents for the robotic devices used in this work. He holds equity positions in Bionik Laboratories, the company that manufactures this type of technology under license to MIT. All other authors declare that they have no competing interests.

\section{Publisher's Note}

Springer Nature remains neutral with regard to jurisdictional claims in published maps and institutional affiliations.

\section{Author details}

${ }^{1}$ Stroke Research Group, Institute of Neuroscience, Newcastle University, 3-4 Claremont Terrace, Newcastle upon Tyne NE2 4AE, UK. ${ }^{2}$ Stroke Northumbria, Northumbria Healthcare NHS Foundation Trust, North Tyneside General Hospital, Rake Lane, North Shields, Tyne and Wear NE29 8NH, UK. ${ }^{3}$ Institute of Health and Society, Newcastle University, Baddiley-Clark Building, Richardson Road, Newcastle upon Tyne NE2 4AX, UK. ${ }^{4}$ Barking, Havering and Redbridge University Hospitals NHS Trust Queen's Hospital, Rom Valley Way, Romford, Essex RM7 OAG, UK. ${ }^{5}$ North West London Hospitals NHS Trust, Northwick Park Hospital, Watford Road, Harrow HA1 3UJ, UK. 'University of Glasgow, Queen Elizabeth University Hospital, 1342 Govan Road, Govan, Glasgow G51 4TF, UK. 'Department of Child Health, Institute of Neuroscience, Newcastle University, Royal Victoria Infirmary, Queen Victoria Road, Newcastle upon Tyne NE1 4LP, UK. ${ }^{8}$ Medical Sciences Division, University of Oxford, and Oxford University Hospitals NHS Foundation Trust, Oxford OX3 9DU, UK. ${ }^{9}$ Oxford Academic Health Science Network, Magdalen Centre North Oxford Science Business Park, Oxford OX4 4GA, UK. ${ }^{10}$ Health Economics Group, Institute of Health and Society, Newcastle University, Baddiley-Clark Building, Richardson Road, Newcastle upon Tyne NE2 4AX, UK. ${ }^{11}$ Contact via: Stroke Research Group, Institute of Neuroscience, Newcastle University, 3-4 Claremont Terrace, Newcastle upon Tyne NE2 4AE, UK. ${ }^{12} \mathrm{NHS}$ Greater Glasgow and Clyde, Queen Elizabeth University Hospital, 1342 Govan Road, Govan, Glasgow G51 4TF, UK. ${ }^{13}$ Massachusetts Institute of Technology, 77 Massachusetts Avenue, 3-137, Cambridge, MA 02139, USA. ${ }^{14}$ Stroke Northumbria, Northumbria Healthcare NHS Foundation Trust, Wansbeck General Hospital, Woodhorn Lane, Ashington, Northumberland NE63 9JJ, UK. ${ }^{15}$ Institute of Neuroscience, Newcastle University, Clinical Ageing Research Unit, Campus for Ageing and Vitality, Newcastle upon Tyne NE4 5PL, UK. ${ }^{16}$ University of East London, School of Health, Sport and Biosciences, Stratford Campus, Water Lane, Stratford, London E15 4LZ, UK. ${ }^{17}$ Cambridge University Health Partners (Addenbrooke's Hospital), R3 Neurosciences, Addenbrooke's Hospital, Hills Road, Box 83, Cambridge CB2 2QQ, UK. ${ }^{18}$ Institute for Applied Health Research and School of Health and Life Sciences, Glasgow Caledonian University, Cowcaddens Road, Glasgow G4 OBA, UK. ${ }^{19}$ Department of Pharmacy, Health and Wellbeing, Faculty of Applied Sciences, Science Complex, University of Sunderland, City Campus, Chester Road, Sunderland SR1 3SD, UK.

Received: 15 October 2016 Accepted: 4 July 2017

Published online: 20 July 2017

\section{References}

1. Adamson J, Beswick A, Ebrahim S. Is stroke the most common cause of disability? J Stroke Cerebrovasc Dis. 2004;13:171-7.

2. Nakayama $\mathrm{H}$, Jorgensen $\mathrm{HS}$, Raaschou HO, Olsen TS. Recovery of upper extremity function in stroke patients: the Copenhagen Stroke Study. Arch Phys Med Rehabil. 1994;75:394-8.

3. Kwakkel G, Kollen BJ, van der Grond J, Prevo AJ. Probability of regaining dexterity in the flaccid upper limb: impact of severity of paresis and time since onset in acute stroke. Stroke. 2003;34:2181-6.

4. Pollock A, St George B, Fenton M, Firkins L. Top 10 research priorities relating to life after stroke-consensus from stroke survivors, caregivers, and health professionals. Int J Stroke. 2014;9:313-20.

5. Hallett M. Plasticity in the human motor system. Neuroscientist. 1999;5:324-32.

6. Hebb DO. The organization of behavior. New York: Wiley; 1949

7. Pollock A, Farmer SE, Brady MC, Langhorne P, Mead GE, Mehrholz J, van Wijck F. Interventions for improving upper limb function after stroke. Cochrane Database Syst Rev. 2014;11, CD010820.

8. Mehrholz J, Pohl M, Platz T, Kugler J, Elsner B. Electromechanical and robotassisted arm training for improving activities of daily living, arm function, and arm muscle strength after stroke. Cochrane Database Syst Rev. 2015;11, CD006876.

9. Norouzi-Gheidari N, Archambault PS, Fung J. Effects of robot-assisted therapy on stroke rehabilitation in upper limbs: systematic review and meta-analysis of the literature. J Rehabil Res Dev. 2012;49:479-96.

10. Chan AW, Tetzlaff JM, Altman DG, Laupacis A, Gotzsche PC, Krleza-Jeric K, Hrobjartsson A, Mann H, Dickersin K, Berlin JA, et al. SPIRIT 2013 statement: defining standard protocol items for clinical trials. Ann Intern Med. 2013:158:200-7.

11. Lyle RC. A performance test for assessment of upper limb function in physical rehabilitation treatment and research. Int J Rehabil Res. 1981;4:483-92

12. Brott T, Adams Jr HP, Olinger CP, Marler JR, Barsan WG, Biller J, Spilker J, Holleran R, Eberle R, Hertzberg $V$, et al. Measurements of acute cerebral infarction: a clinical examination scale. Stroke. 1989;20:864-70.

13. Montreal Cognitive Assessment (MoCA) Administration and Scoring Instructions http://www.mocatest.org/. Accessed 1 July 2016

14. Al-Khawaja I, Wade DT, Collin CF. Bedside screening for aphasia: a comparison of two methods. J Neurol. 1996;243:201-4.

15. Fugl-Meyer AR, Jaasko L, Leyman I, Olsson S, Steglind S. The post-stroke hemiplegic patient. 1. a method for evaluation of physical performance. Scand J Rehabil Med. 1975:7:13-31.

16. Collin C, Wade DT, Davies S, Horne V. The Barthel ADL Index: a reliability study. Int Disabil Stud. 1988;10:61-3.

17. Mahoney Fl, Barthel DW. Functional evaluation: the Barthel Index. MD State Med J. 1965;14:61-5.

18. Williams A. The EuroQol Instrument. The Netherlands: Springer; 2005.

19. Price DD, Bush FM, Long S, Harkins SW. A comparison of pain measurement characteristics of mechanical visual analogue and simple numerical rating scales. Pain. 1994;56:217-26.

20. Beecham J, Knapp M. Costing psychiatric interventions. In: Thornicroft G editor. Measuring mental health needs. 2nd ed. London: Gaskell; 2001.

21. Forster A, Dickerson J, Young J, Patel A, Kalra L, Nixon J, Smithard D, Knapp M, Holloway I, Anwar S, et al. A structured training programme for caregivers of inpatients after stroke (TRACS): a cluster randomised controlled trial and cost-effectiveness analysis. Lancet. 2013;382:2069-76.

22. Patel A, Knapp M, Evans A, Perez I, Kalra L. Training care givers of stroke patients: economic evaluation. BMJ. 2004;328:1102

23. Krebs HI, Hogan N, Aisen ML, Volpe BT. Robot-aided neurorehabilitation. IEEE Trans Rehabil Eng. 1998;6:75-87.

24. Krebs HI, Volpe BT, Williams D, Celestino J, Charles SK, Lynch D, Hogan N. Robot-aided neurorehabilitation: a robot for wrist rehabilitation. IEEE Trans Neural Syst Rehabil Eng. 2007;15:327-35.

25. Masia L, Krebs HI, Cappa P, Hogan N. Design and characterization of hand module for whole-arm rehabilitation following stroke. IEEE ASME Trans Mechatron. 2007:12:399-407.

26. Hoffmann TC, Glasziou PP, Boutron I, Milne R, Perera R, Moher D, Altman DG, Barbour V, Macdonald $H$, Johnston M, et al. Better reporting of interventions: template for intervention description and replication (TIDieR) checklist and guide. BMJ. 2014;348:g1687.

27. Shaw L, Rodgers H, Price C, van Wijck F, Shackley P, Steen N, Barnes M, Ford G, Graham L. BoTULS: a multicentre randomised controlled trial to evaluate the clinical effectiveness and cost-effectiveness of treating upper limb spasticity due to stroke with botulinum toxin type A. Health Technol Assess. 2010;14:1-113. iii-iv.

28. Shaw LC, Price Cl, van Wijck FM, Shackley P, Steen N, Barnes MP, Ford GA, Graham LA, Rodgers H. Botulinum Toxin for the Upper Limb after Stroke (BoTULS) Trial: effect on impairment, activity limitation, and pain. Stroke. 2011;42:1371-9.

29. van Wijck F. PhD Thesis. Skill acquisition in people with chronic upper limb spasticity after stroke. Queen Margaret University, Edinburgh, 2006. http:// ethos.bl.uk/OrderDetails.do?uin=uk.bl.ethos.429770. Accessed 1 July 2016.

30. Brkic L, Shaw L, van Wijck F, Price C, Watkins C, Forster A, Langhorne P, Rodgers $\mathrm{H}$. Repetitive arm functional tasks after stroke (RAFTAS): a pilot randomised controlled trial. Pilot Feasibility Stud. 2016;2:50.

31. National Institute for Health and Clinical Excellence. Stroke Quality Standard. 2012. https://www.nice.org.uk/guidance/qs2/chapter/Quality-statement-2Intensity-of-stroke-rehabilitation Accessed 1 July 2016.

32. Intercollegiate Stroke Working Party. National Sentinel Stroke Clinical Audit. London: Royal College of Physicians; 2015.

33. Duncan PW, Bode RK, Min Lai S, Perera S. Rasch analysis of a new strokespecific outcome scale: the Stroke Impact Scale. Arch Phys Med Rehabil. 2003;84:950-63. 
34. Glazener C, Boachie C, Buckley B, Cochran C, Dorey G, Grant A, Hagen S, Kilonzo M, McDonald A, McPherson G, et al. Conservative treatment for urinary incontinence in Men After Prostate Surgery (MAPS): two parallel randomised controlled trials. Health Technol Assess. 2011:15:1-290. iii-iv.

35. Hockenhull JC, Dwan K, Boland A, Smith G, Bagust A, Dundar Y, Gamble C, McLeod C, Walley T, Dickson R. The clinical effectiveness and costeffectiveness of central venous catheters treated with anti-infective agents in preventing bloodstream infections: a systematic review and economic evaluation. Health Technol Assess. 2008;12:iii-iv. xi-xii, 1-154.

36. Progress and Safety Reporting: National Research Ethics Committee (NRES) guidance for non CTIMP trials http://www.hra.nhs.uk/resources/during-andafter-your-study/progress-and-safety-reporting/. Accessed 1 July 2016.

37. van der Lee JH, Wagenaar RC, Lankhorst GJ, Vogelaar TW, Deville WL, Bouter LM. Forced use of the upper extremity in chronic stroke patients: results from a single-blind randomized clinical trial. Stroke. 1999:30:2369-75.

38. Curtis L. Unit costs of health and social care. Canterbury: PSSRU: University of Kent; 2007.

39. Drummond M, O'Brien B, Stoddart G, Torrance G. Methods for the economic evaluation of health care programmes. 3rd ed. Oxford: Oxford University Press; 2005.

40. Devlin N, Shah K, Feng Y, Mulhern B, van Hout B. Valuing health-related quality of life: an EQ-5D-5L value set for England. In: Office of Health Economics, Research paper 16/01. London: Office of Health Economics Publications; 2016

41. Fenwick E, Byford S. A guide to cost-effectiveness acceptability curves. Br J Psychiatry. 2005:187:106-8.

42. Philips Z, Ginnelly L, Sculpher M, Claxton K, Golder S, Riemsma R, Woolacoot $\mathrm{N}$, Glanville J. Review of guidelines for good practice in decision-analytic modelling in health technology assessment. Health Technol Assess. 2004;8: iii-iv. ix-xi, 1-158.

43. May C, Finch T. Implementation, embedding and integration: an outline of Normalization Process Theory. Sociology. 2009;43:535-54.

44. Glaser BG, Straus AL. The discovery of grounded theory: strategies for qualitative research. Chicago: Aldine Publishers; 1967.

45. Department of Health. Research governance framework for health and social care. 2nd ed. 2005.

46. Langhorne P, Coupar F, Pollock A. Motor recovery after stroke: a systematic review. Lancet Neurol. 2009;8:741-54.

47. Wade DT. Goal setting in rehabilitation: an overview of what, why and how Clin Rehabil. 2009;23:291-5.

\section{Submit your next manuscript to BioMed Central and we will help you at every step:}

- We accept pre-submission inquiries

- Our selector tool helps you to find the most relevant journal

- We provide round the clock customer support

- Convenient online submission

- Thorough peer review

- Inclusion in PubMed and all major indexing services

- Maximum visibility for your research

Submit your manuscript at www biomedcentral.com/submit 